\title{
Right aortic arch aortopulmonary window associated with left pulmonary artery originate from patent ductus arteriosus
}

\author{
Qiang Fan ${ }^{1}$, Yunfei Ling ${ }^{1}$, Yue Wang ${ }^{1}$, and Qi $\mathrm{An}^{1}$ \\ ${ }^{1}$ Sichuan University West China Hospital
}

September 16, 2020

\begin{abstract}
Aortopulmonary window (APW) is a rare but serious congenital cardiac malformation, most patients with APW will die from congestive heart failure a few months after birth. However, in this case we presented is an extremely rare condition that consist of a type III APW and a ductus arteriosus originated left pulmonary artery. Preoperative diagnosis included echocardiography and chest computerized tomography revealed anatomical structure of the heart and great vessel clearly, cardiac catheterization indicated that the pulmonary resistances indices were 2.92 wood $\mathrm{U} / \mathrm{m}^{\wedge} 2$ in $\mathrm{LPA}$ and 3.35 wood $\mathrm{U} / \mathrm{m} \wedge 2$ in RPA, Qp:Qs was 3.26. This patient underwent surgical correction at the age of 9 and successfully survived.
\end{abstract}

Right aortic arch aortopulmonary window associated with left pulmonary artery originate from patent ductus arteriosus

Qiang Fan* MD, Yunfei Ling* PhD, Yue Wang, Qi An\# MD

Department of Cardiovascular surgery, West China Hospital, Sichuan University

* These authors contributed equally to this work and should be considered co-first authors

\# Corresponding author information: Tel: +86 28 85422897, Fax: +86 28 85422897, E-mail: anqi@scu.edu.cn, Add: No. 37 Guo Xue Xiang, Chengdu, Sichuan 610041, People’s Republic of China.

Keywords : aortopulmoanry window, patent ducts arteriosus, anomalous origin of left pulmonary artery

Funding : none.

Abstract Aortopulmonary window (APW) is a rare but serious congenital cardiac malformation, most patients with APW will die from congestive heart failure a few months after birth. However, in this case we presented is an extremely rare condition that consist of a type III APW and a ductus arteriosus originated left pulmonary artery. Preoperative diagnosis included echocardiography and chest computerized tomography revealed anatomical structure of the heart and great vessel clearly, cardiac catheterization indicated that the pulmonary resistances indices were 2.92 wood $\frac{U}{m^{2}}$ in LPA and 3.35 wood $\frac{U}{m^{2}}$ in RPA, $Q_{p}: Q_{s}$ was 3.26 . This patient underwent surgical correction at the age of 9 and successfully survived.

A 9-year-old girl was referred to our department with significant fatigue and shortness of breath after exercise but without cyanosis and clubbed fingers, the body weight and height was lower than those of the same age. Physical examination showed the heart border was expanded and heat murmur was noted over the left side of the chest. Electrocardiogram recorded a heart rate of 88 beats per minutes and indicated a sinus arrhythmia and high left ventricular voltage. Chest x-ray showed mild pulmonary congestion, severe cardiomegaly and right hilum convergence sign (panel A). The echocardiography revealed a right aortic arch and suggested a type III aortopulmonary window (APW), the right pulmonary artery (RPA) had a proximal origin from posterior part of the ascending aorta (panel B), a patent ductus arteriosus (PDA) originated 
from brachiocephalic artery (panel C), but the left pulmonary artery (LPA) was not noted. Computerize tomography (CT) scan of chest was performed, type III APW and anomalous origin of RPA was confirmed (panel D) while the LPA was found to originated from PDA with a stenosed orifice (panel E). The threedimensional reconstruction of the CT scan revealed the relationship between aorta and RPA and LAP clearly (panel $\mathrm{G}$ and $\mathrm{H}$ ). Cardiac catheterization was performed preoperatively revealed that the small connection between PDA and LPA (panel F), the measured pulmonary resistances indices were 2.92 wood $\frac{U}{m^{2}}$ in LPA and 3.35 wood $\frac{U}{m^{2}}$ in RPA, $Q_{p}: Q_{s}$ was 3.26 . The patient then underwent open-heart surgery under cardiopulmonary bypass, intraoperative observation was coincident with the preoperative diagnosis (panel I). This patient stayed in ICU for 8 days and discharged unevenfully 13 days after surgery.

Consent The study were approved by the relevant ethics committees, and oral informed consent was obtained for the participant.

Figure legend : (A) Chest X-ray showed pulmonary congestion, severe cardiomegaly and right hilum convergence sign. (B) Echocardiography showed APW and the RPA. (C) Echocardiography showed PDA (red arrow). (D) Chest CT showed APW and RPA. (E) Chest CT showed LPA originated from PDA (red arrow). (F) Cardiac catheterization revealed the small connection between PDA (red arrow) and LPA. (G) and $(\mathrm{H})$ showed the relationship between Ao and RPA and LAP. (I) Intraoperative observation was coincident with the preoperative diagnosis. $A o$, ascending aorta; $P A$, pulmonary artery; $R P A$, right pulmonary artery; $L P A$, left pulmonary artery; $P D A$, patent ductus arteriosus.
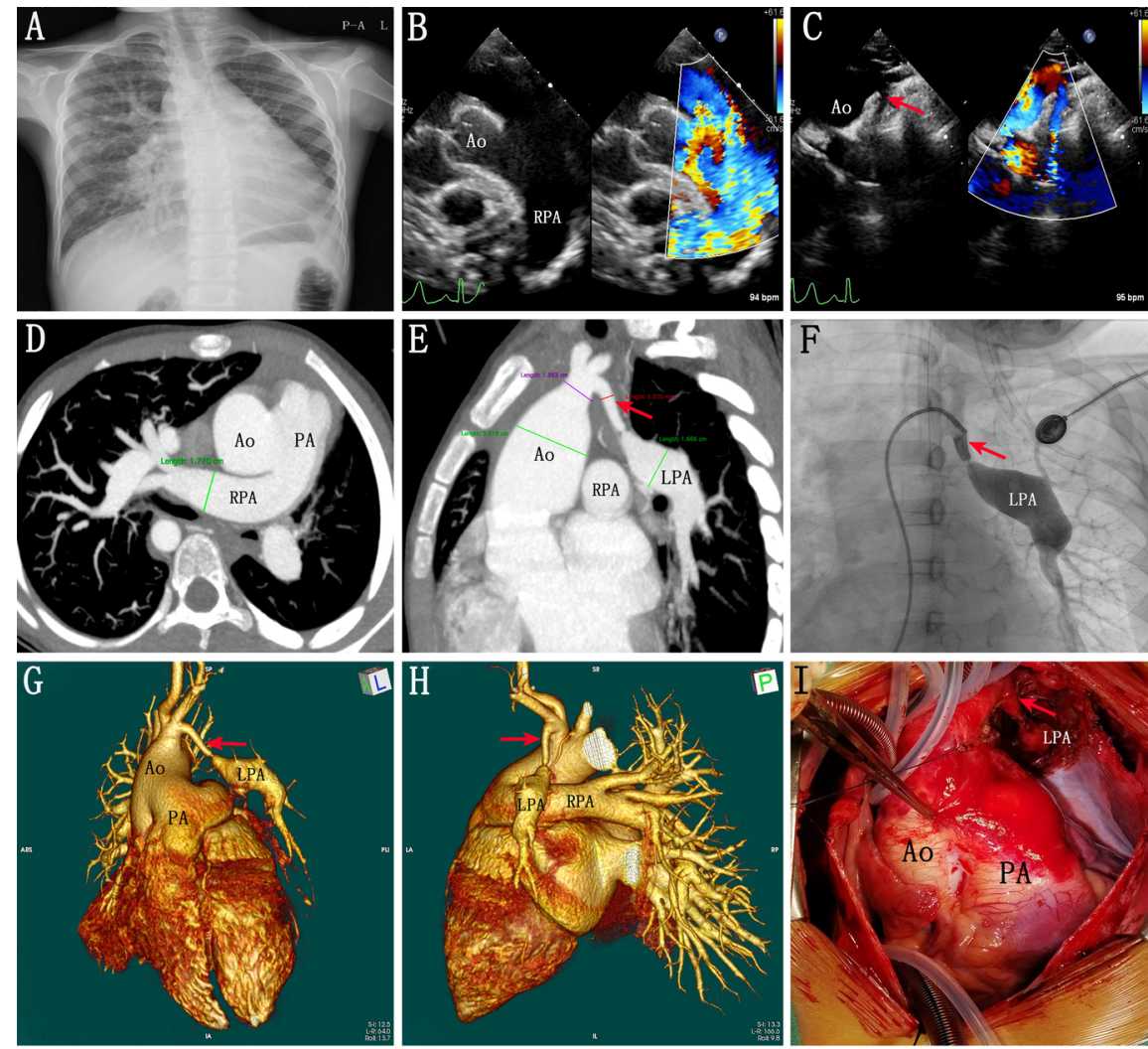